\title{
TATE COHOMOLOGY OF CONNECTED K-THEORY FOR ELEMENTARY ABELIAN GROUPS REVISITED
}

\author{
PO HU, IGOR KRIZ AND PETR SOMBERG
}

\begin{abstract}
Tate cohomology (as well as Borel homology and cohomology) of connective K-theory for $G=(\mathbb{Z} / 2)^{n}$ was completely calculated by Bruner and Greenlees [2]. In this note, we essentially redo the calculation by a different, more elementary method, and we extend it to $p>2$ prime. We also identify the resulting spectra, which are products of Eilenberg-Mac Lane spectra, and finitely many finite Postnikov towers. For $p=2$, we also reconcile our answer completely with the result of [2, which is in a different form, and hence the comparison involves some non-trivial combinatorics.
\end{abstract}

\section{IntRoduction}

Tate cohomology of finite groups was first discovered in number theory, where it was noticed that in some statements related to duality, the 0'th cohomology has to be "corrected" by factoring out the image of the norm map from 0'th homology (see for example Serre [12]). Considering also the kernel of the norm map, finite group homology and cohomology, with the 0'th groups "corrected", fit into one distinguished theory which became known as Tate cohomology.

In stable homotopy theory, the natural question was to find an appropriate definition of Tate cohomology for generalized cohomology theories with a finite (or compact Lie) group action, i.e. equivariant spectra [10]. This was accomplished in the fundamental paper [6] by Greenlees and May. Generalized Tate cohomology has since become an important tool in stable homotopy theory, notably in [7, 9, 8]. Generalized, in contrast with ordinary, Tate cohomology is a key tool for understanding completion theorems, which relate, by completion, the coefficients of an equivariant cohomology theory to its Borel cohomology. The first known completion theorem was proved by Atiyah and Segal for K-theory [1]. Other important cases discovered since then include the Segal conjecture, proved by G.Carlsson [3], and a completion

The authors acknowledge support by grant GA CR P201/12/G028. Kriz also acknowledges the support of a Simons Collaboration Grant. 
theorem for complex cobordism by Greenlees and May [7]. This aspect of Tate cohomology is not visible at all on the original concept of ordinary Tate cohomology simply because ordinary cohomology does not satisfy a completion theorem.

The role of generalized Tate cohomology in completion theorems stems from the fact that it is, like in the ordinary case, the cofiber of a norm map [6] from the Borel homology to the Borel cohomology of a given equivariant spectrum. All these constructions forget much of the equivariant structure, in the sense that a morphism of equivariant spectra, which is an equivalence non-equivariantly, induces an isomorphism on them. This aspect of Tate cohomology was, more recently, used in a different context by Nikolaus and Scholze [11] to simplify much of the theory of topological cyclic homology.

In the case of K-theory, the Atiyah-Segal completion theorem [1] was reinteterpreted by Greenlees and May [6] to say that the Tate cohomology of K-theory is rational. In connection with this fact they asked about the structure of Tate cohomology of connective K-theory (in the complex and orthogonal cases), and proposed an answer in terms of a certain part of K-theory Tate cohomology. In particular, they conjectured that it is a wedge sum of Eilenberg-MacLane spectra [4], Conjecture 13.4.

Bruner and Greenlees [2] computed Borel k-homology, Borel k-cohomology and Tate k-cohomology for the group $G=(\mathbb{Z} / 2)^{r}$ for $r>1$. They disproved the Greenlees-May conjecture, showing, however, that a closely related statement holds for $G=(\mathbb{Z} / 2)^{r}$. In particular, there is a large summand which is a sum of suspensions of $H \mathbb{Z} / 2$, which is essentially dual in k-Borel homology and cohomology, and both duals are present in Tate cohomology. The computation of [2] is non-trivial, using techniques of local algebra, as well as the Adams spectral sequence. The non-torsion part is also closely related to what was conjectured by Greenlees and May in that it is a sum of copies of $H \mathbb{Z}_{2}$, and finitely many finite Postnikov towers.

The main purpose of this note is to exhibit a different approach to these calculations, using more direct and elementary methods. We also extend the calculations to $p>2$. Essentially, we directly use induction on $r$, smashing (or applying the function spectrum) to one additional copy of $B \mathbb{Z} / p_{+}$at a time, exploiting the fact that most of the $k$-modules involved have Ext-dimension $\leq 1$ (noticing that having this on coefficients implies the same statement in spectral algebra). Our method 
gives a closed formulas for the Poincare series of the torsion part of the calculation. For $p=2$, we prove that this is in fact the same answer as the result of [2], which is a non-trivial combinatorial calculation.

The present paper is organized as follows. We treat the $p=2$ case first, since it is simpler, and we know it first. In Section 2, we compute the $k$-Borel homology and cohomology for $p=2$, essentially using homological algebra, which in this case nicely translates to homological algebra of $k$-modules. In Section 3 , we compute the norm map for $p=2$, and identify the $k$-Tate cohomology for $p=2$ in Theorem [11. In Section 4, we explain what needs to be added to those constructions for handling the case $p>2$. The computation of $k$-Tate cohomology is presented in Theorem 21. In Section 5, we give the explicit algebraic comparison of our result for $p=2$ with the result of [2].

\section{Borel $k$-HOMOlogy And COHOMOlogy of $(\mathbb{Z} / 2)^{i}$}

Let $k$ denote connective complex K-theory $E_{\infty}$ ring spectrum. We have $k_{*}=\mathbb{Z}[\beta]$ where $\beta$ is the Bott element in degree 2 . Consider the $\mathbb{Z}[\beta]$-modules

$$
N=\mathbb{Z}\left[\frac{\beta}{2}\right], M=N / \mathbb{Z}[\beta] .
$$

We shall write Tor for $\operatorname{Tor}^{\mathbb{Z}[\beta]}$. For any graded $\mathbb{Z}[\beta]$-module $W$, and a power series $p(w)=\sum_{n} a_{n} w^{n} \in \mathbb{N}_{0}\left[\left[w, w^{-1}\right]\right]$, we shall denote by $W p(w)$ a sum of copies of shifts of $W$ where there are $a_{n}$ copies of $W[n]$ (i.e. of $W$ shifted up by $n \in \mathbb{Z}$ ).

Lemma 1. (1) For every $\mathbb{Z}[\beta]$-module $W$, we have

$$
\operatorname{Tor}_{\geq 2}(M, W)=\operatorname{Tor}_{\geq 2}(N, W)=0 \text {. }
$$

(2) We have

$$
N \otimes_{\mathbb{Z}[\beta]} N \cong N \oplus \mathbb{Z} / 2 \frac{w^{2}}{\left(1-w^{2}\right)^{2}}, \operatorname{Tor}_{1}(N, N)=0
$$

(Note that the graded module $\mathbb{Z} / 2$ must have trivial action of $\beta$.

(3) We have

$$
N \otimes_{\mathbb{Z}[\beta]} M \cong \mathbb{Z} / 2 \frac{w^{2}}{\left(1-w^{2}\right)^{2}}, \operatorname{Tor}_{1}(M, N)=0 .
$$

(4) We have

$$
M \otimes_{\mathbb{Z}[\beta]} M \cong \mathbb{Z} / 2 \frac{w^{4}}{\left(1-w^{2}\right)^{2}}, \operatorname{Tor}_{1}(M, M) \cong M[2] .
$$


Proof. We have a free $\mathbb{Z}[\beta]$-resolution of $N$ of the form

$$
0 \rightarrow \bigoplus_{i \geq 1} \mathbb{Z}[\beta]\left\{t_{i}\right\} \rightarrow \bigoplus_{i \geq 0} \mathbb{Z}[\beta]\left\{z_{i}\right\}
$$

where $\left|z_{i}\right|=\left|t_{i}\right|=2 i$,

$$
t_{i} \mapsto 2 z_{i}-\beta z_{i-1}, i \geq 1 .
$$

The augmentation sends

$$
z_{i} \mapsto\left(\frac{\beta}{2}\right)^{i}
$$

Similarly, we have a free $\mathbb{Z}[\beta]$-resolution of $M$ of the form

$$
0 \rightarrow \bigoplus_{i \geq 0} \mathbb{Z}[\beta]\left\{t_{i}\right\} \rightarrow \bigoplus_{i \geq 0} \mathbb{Z}[\beta]\left\{z_{i}\right\}
$$

with (2) and

$$
t_{0} \mapsto z_{0} .
$$

Statement 1 follows.

To prove Statement 2, apply ? $\otimes_{\mathbb{Z}[\beta]} N$ to (11). We obtain

$$
\begin{gathered}
\bigoplus_{i \geq 1} \mathbb{Z}\left[\frac{\beta}{2}\right]\left\{t_{i}\right\} \rightarrow \bigoplus_{i \geq 0} \mathbb{Z}\left[\frac{\beta}{2}\right]\left\{z_{i}\right\}, \\
t_{i} \mapsto 2\left(z_{i}-\frac{\beta}{2} z_{i-1}\right), i \geq 1 .
\end{gathered}
$$

Clearly, this is injective and the cokernel is

$$
\mathbb{Z}\left[\frac{\beta}{2}\right]\left\{z_{0}\right\} \oplus \bigoplus_{i \geq 1} \mathbb{Z} / 2\left[\frac{\beta}{2}\right]\left\{z_{i}-\frac{\beta}{2} z_{i-1}\right\},
$$

as claimed.

For Statement 3, consider the short exact sequence of $\mathbb{Z}[\beta]$-modules

$$
0 \rightarrow \mathbb{Z}[\beta] \rightarrow N \rightarrow M \rightarrow 0,
$$

and the associated long exact sequence of $\operatorname{Tor}(?, N)$. We get

$$
0 \longrightarrow \operatorname{Tor}_{1}(M, N) \longrightarrow N \stackrel{\alpha}{\longrightarrow} N \oplus \mathbb{Z} / 2 \frac{w^{2}}{\left(1-w^{2}\right)^{2}} \longrightarrow N \otimes_{\mathbb{Z}[\beta]} M \longrightarrow 0
$$

where $\alpha$ is the inclusion of the first summand. Statement 3 follows.

For Statement 4, consider the long exact sequence obtained by applying $\operatorname{Tor}(?, M)$ to (5). We obtain

$$
0 \longrightarrow \text { Tor }_{1}(M, M) \longrightarrow M \stackrel{\gamma}{\longrightarrow} \mathbb{Z} / 2 \frac{w^{2}}{\left(1-w^{2}\right)^{2}} \longrightarrow M \otimes_{\mathbb{Z}[\beta]} M \longrightarrow 0
$$

The map $\gamma$ is the identity on

$$
\bigoplus_{i \geq 1} \mathbb{Z} / 2\left\{z_{i}\right\}
$$


and 0 otherwise. Realizing that

$$
\operatorname{Ker}(\gamma) \cong M[2]
$$

Statement 4 follows.

We now turn to discussing $k$-module structures. For an $E_{\infty}$-ring spectrum $R$, by a module, we shall always mean an $E_{\infty}$-module. For background information about how to do commutative algebra over $E_{\infty}$-ring spectra, the reader is referred to [5].

By a degree $0,1-R_{*}$-resolution we shall mean a free resolution of an $R_{*}$-module non-trivial only in degrees 0,1 . It is easy to see that a degree $0,1-R_{\star}$-resolution is realized by an $R$-module uniquely up to $R$-module equivalence.

Thus, we have unique $k$-modules $\mathscr{M}, \mathscr{N}$ with $\mathscr{M}_{\star}=M, \mathscr{N}_{\star}=N$ and, furthermore,

$$
k \wedge B \mathbb{Z} / 2 \sim \mathscr{M}[-1]
$$

as $k$-modules. Letting

$$
H=k /(\beta, 2)
$$

(recall that the order of killing elements does not matter), we further conclude from Lemma 1 that

$$
\mathscr{M} \wedge_{k} \mathscr{N} \sim H \frac{w^{2}}{\left(1-w^{2}\right)^{2}}=H w^{2}\left(\sum_{i=0}^{\infty} w^{2 i}\right)^{2}=H \sum_{i=1}^{\infty} i w^{2 i}
$$

(using the same convention for module spectra as for graded modules). This is because we get a map to $H[2(i+j+1)]$ by sending the generator $z_{i+1}(\beta / 2)^{j}$ to $H$, while sending the generators $z_{k}(\beta / 2)^{\ell}$ to 0 for $(k, \ell) \neq$ $(i+1, j)$.

Now consider the cofibration sequence

$$
\mathscr{M} \rightarrow \mathscr{N} \wedge_{k} \mathscr{M} \rightarrow \mathscr{M} \wedge_{k} \mathscr{M}
$$


From the above discussion, we obtain a commutative diagram of cofibrations in the derived category of $k$-modules

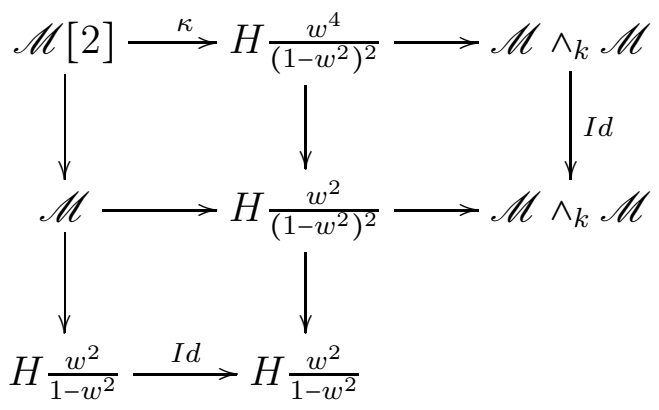

where the map $\kappa$ is 0 on coefficients. But it follows from the resolution of $\mathscr{M}$ that all non-trivial maps of $k$-modules $\mathscr{M} \rightarrow H[2 i]$ are non-zero on coefficients. Thus, $\kappa=0$, and we have proved

Proposition 2. As k-modules, we have

$$
k \wedge B \mathbb{Z} / 2 \wedge B \mathbb{Z} / 2[2] \sim \mathscr{M} \wedge_{k} \mathscr{M} \sim \mathscr{M}[3] \vee H \frac{w^{4}}{\left(1-w^{2}\right)^{2}} .
$$

To go farther, we note the following:

Lemma 3. We have

$$
H \wedge_{k} \mathscr{M} \sim H \frac{w^{2}}{1-w} .
$$

Proof. Apply $? \wedge_{k} H$ to the geometric realization of (3). We see that we can eliminate the $i=0$ terms, since they are linked by an isomorphism. Omitting those terms, we claim that the resulting map of $k$-modules

$$
H \frac{w^{2}}{1-w^{2}} \rightarrow H \frac{w^{2}}{1-w^{2}}
$$

is zero. To this end, it suffices to show that the self-maps $2, \beta$ of $H$ are both zero. But considering the defining resolution of $H$ by free $k$-modules, we see that the only non-zero self-maps of $H$ as $k$-modules are in negative degrees.

Let

$$
E=H \frac{w^{4}}{\left(1-w^{2}\right)^{2}}
$$

be the "error term" in Proposition 2 ,

Proposition 4. The following statements hold as $k$-modules. 
(1) For $i \geq 0$,

$$
E \wedge_{k} \underbrace{\mathscr{M} \wedge_{k} \cdots \wedge_{k} \mathscr{M}}_{i \text { times }}=H \frac{w^{2(i+2)}}{\left(1-w^{2}\right)^{2}(1-w)^{i}} .
$$

(2) For $i \geq 2$,

$$
\begin{aligned}
& k \wedge \underbrace{B \mathbb{Z} / 2 \wedge \cdots \wedge B \mathbb{Z} / 2}_{i \text { times }}[i]=\underbrace{\mathscr{M} \wedge_{k} \cdots \wedge_{k} \mathscr{M}}_{i \text { times }}= \\
& \mathscr{M}[3(i-1)] \vee H \frac{w^{4}}{\left(1-w^{2}\right)^{2}}\left(\frac{w^{2}}{1-w}\right)^{i-2}\left(\frac{(1-w)^{i-1} w^{i-1}-1}{(1-w) w-1}\right) .
\end{aligned}
$$

(3) For $i \geq 0$,

$$
\begin{aligned}
& k \wedge B(\mathbb{Z} / 2)_{+}^{i}=k \vee \mathscr{M} w^{-3}\left(\left(1+w^{2}\right)^{i}-1\right) \vee \\
& H \frac{1}{\left(1-w^{2}\right)^{2}(1-w)^{i-1}}\left(\frac{1-\left(1+w^{2}\right)^{i}(1-w)^{i}}{1-\left(1+w^{2}\right)(1-w)}-\frac{1-(1-w)^{i}}{1-(1-w)}\right) .
\end{aligned}
$$

Proof. We have $k \wedge B \mathbb{Z} / 2_{+}=k \vee \Sigma^{-1} \mathscr{M}$. Statement 1 follows immediately from Lemma 3. Statement 2 follows from Proposition 2 and Lemma 3 by induction, using the formula

$$
1+\frac{1}{a}+\cdots+\frac{1}{a^{i-2}}=\frac{a^{i-1}-1}{(a-1) a^{i-2}} .
$$

To prove Statement 3, let, as an induction hypothesis,

$$
k \wedge B(\mathbb{Z} / 2)_{+}^{i}=k \vee \mathscr{M} \cdot p_{i} \vee H \cdot q_{i} .
$$

We have proved that $p_{1}=w^{-1}, q_{1}=0$, and

$$
\begin{aligned}
& k \vee \mathscr{M} \cdot p_{i+1} \vee H \cdot q_{i+1}= \\
& \left(k \vee \mathscr{M} \cdot p_{i} \vee H \cdot q_{i}\right) \wedge_{k}\left(k \vee \mathscr{M} w^{-1}\right)= \\
& k \vee \mathscr{M} \cdot\left(w^{-1}+\left(1+w^{2}\right) p_{i}\right) \vee H \cdot\left(\frac{w^{3}}{\left(1-w^{2}\right)^{2}} p_{i}+\frac{q_{i}}{1-w}\right) .
\end{aligned}
$$

Thus, we have

$$
\begin{gathered}
p_{i+1}=w^{-1}+\left(1+w^{2}\right) p_{i}, \\
q_{i+1}=\frac{w^{3}}{\left(1-w^{2}\right)^{2}} p_{i}+\frac{q_{i}}{1-w} .
\end{gathered}
$$

From (9), we obtain

$$
p_{i}=\left(\left(1+w^{2}\right)^{i}-1\right) w^{-3},
$$


so (10) gives

$$
q_{i+1}=\frac{\left(1+w^{2}\right)^{i}-1}{\left(1-w^{2}\right)^{2}}+\frac{q_{i}}{1-w} .
$$

Solving the recursion gives Statement 3 .

We now calculate $F\left(B(\mathbb{Z} / 2)_{+}^{i}, k\right)$. Again, the restriction map $B \mathbb{Z} / 2_{+} \rightarrow$ $S^{0}$ induces a splitting

$$
F\left(B \mathbb{Z} / 2_{+}, k\right) \sim k \vee F(B \mathbb{Z} / 2, k) .
$$

Let

$$
\mathscr{P}=F(B \mathbb{Z} / 2, k)=x k[[x]] /(x \beta+2)
$$

where

$$
k[[x]]=\prod_{i \geq 0} k\left\{x^{i}\right\},|x|=-2 .
$$

This can be expressed, in an obvious way, as a homotopy limit of $k[x] / x^{j}$ in the obvious sense. When involving these $k$-modules, we shall denote by $\widehat{\lambda}_{k}$ the operation of taking $\wedge_{k}$ on the corresponding "truncated polynomial" modules, and then passing to homotopy limit of the resulting inverse sequence. Thus, similarly to Lemma 1, Proposition 2 and Lemma 3, we prove that

$$
\begin{gathered}
\mathscr{P} \widehat{\wedge}_{k} \mathscr{P} \sim \mathscr{P}[-2] \vee H \frac{w^{-4}}{\left(1-w^{-2}\right)^{2}}, \\
\mathscr{P} \widehat{\wedge}_{k} H \sim H \frac{w^{-1}}{1-w^{-1}} .
\end{gathered}
$$

Analogously to the proof of Proposition 4, we then obtain

Proposition 5. We have

$$
\begin{aligned}
& F\left(B(\mathbb{Z} / 2)^{i}, k\right) \sim \mathscr{P} \cdot\left(\left(1+w^{-2}\right)^{i}-1\right) \vee \\
& H \frac{1}{\left(1-w^{-2}\right)^{2}\left(1-w^{-1}\right)^{i-1}}\left(\frac{1-\left(1+w^{-2}\right)^{i}\left(1-w^{-1}\right)^{i}}{1-\left(1+w^{-2}\right)\left(1-w^{-1}\right)}-\frac{1-\left(1-w^{-1}\right)^{i}}{1-\left(1-w^{-1}\right)}\right) .
\end{aligned}
$$

Note the interesting symmetry of the "error terms" in Borel homology and cohomology. 


\section{Tate Cohomology}

Now to compute the Tate cohomology

$$
\widehat{k}^{(\mathbb{Z} / 2)^{n}},
$$

we use the cofibration sequence of [6]

$$
k \wedge B(\mathbb{Z} / 2)_{+}^{n} \stackrel{N}{\longrightarrow} F\left(B(\mathbb{Z} / 2)_{+}^{n}, k\right) \longrightarrow \widehat{k}^{(\mathbb{Z} / 2)^{n},}
$$

and the computation of Proposition 5, and Statement 3 of Proposition 4. First, note that there is no possibility of extension of $k$-modules involving any of the $H$ summands, since there are no such extensions in non-negative degrees. It follows that (11), as a $k$-module, is a wedge sum of copies of $H$ in degrees we already know, and a $k$-module with coefficients $Q$, which maps, as a $k$-module and in a way injective on coefficients, into $\widehat{K}^{(\mathbb{Z} / 2)^{n}}$, which has coefficients

$$
J_{2}^{\wedge}\left[\beta, \beta^{-1}\right]\left[\frac{1}{2}\right]
$$

(where $J$ denotes the augmentation ideal of $R\left((\mathbb{Z} / 2)^{n}\right)$ and $(?)_{2}^{\wedge}$ means completion at 2$)$. We will see that $Q$ is a completion of a $\mathbb{Z}[\beta]$-module with resolution in degrees 0,1 , and hence, above, the $k$-module is determined by its coefficients $Q$.

To calculate $Q$, let $\alpha_{1}, \ldots, \alpha_{n}$ be $\otimes-\mathbb{Z} / 2$-independent 1-dimensional complex representations of $(\mathbb{Z} / 2)^{n}$. Let $x_{i} \in k^{*}(B \mathbb{Z} / 2)^{n}$ be the Euler class of $\alpha_{i}$. Then we can write $1+x_{i} \beta=\alpha_{i} \in R\left((\mathbb{Z} / 2)^{n}\right)$, and

$$
\widetilde{Q}=J_{2}^{\wedge}\left[\beta, \beta^{-1}\right]\left[\frac{1}{2}\right]=\bigoplus_{\substack{k \geq 1 \\ 1 \leq i_{1}<\cdots<i_{k} \leq n}} \mathbb{Q}_{2}\left[\beta, \beta^{-1}\right]\left\{x_{i_{1}} \ldots x_{i_{k}}\right\} .
$$

It is also worth noting that (13) is, in fact,

$$
\begin{aligned}
& K^{*}\left((B \mathbb{Z} / 2)^{n}\right) / K_{*} \cdot \prod_{i=1}^{n}\left(2-\beta x_{i}\right)= \\
& \mathbb{Z}\left[\beta, \beta^{-1}\right]\left[\left[x_{1}, \ldots, x_{n}\right]\right] /\left(x_{i}\left(2+\beta x_{i}\right), \prod_{i=1}^{n}\left(2+\beta x_{i}\right)\right) .
\end{aligned}
$$

Now $Q$ is a $k^{*}\left((B \mathbb{Z} / 2)^{n}\right)$-submodule of (13), while on a multiple of $x_{i}$, $x_{i}=-\frac{2}{\beta}$. Thus, we have proved

Lemma 6. $Q$ is a $\mathbb{Z}_{2}\left[\beta, \frac{2}{\beta}\right]$-submodule of $\widetilde{Q}$. 
Let $P \subset Q$ be the image of $k^{*} B(\mathbb{Z} / 2)^{n}$. Then we have an extension of $\mathbb{Z}\left[\beta, \frac{2}{\beta}\right]$-modules

$$
0 \rightarrow P \rightarrow Q \rightarrow M \rightarrow 0 .
$$

Proposition 7. The $\mathbb{Z}\left[\beta, \frac{2}{\beta}\right]$-module $P$ is free on generators

$$
\begin{gathered}
x_{i_{1}} \ldots x_{i_{k}}, 1 \leq k<n, i_{1}<\cdots<i_{k}, \\
\prod_{i=1}^{n}\left(1+\frac{\beta}{2} x_{i}\right)-1 .
\end{gathered}
$$

Proof. The fact that the elements (15) are in $P$ follows from the fact that $P$ is the image of $k^{*} B(\mathbb{Z} / 2)^{n}$. For the same reason, it contains 1 , which is equal to (16) by the relation (14). On the other hand, $P$ is generated by the elements (15), (16) $(=1)$, and $x_{1} \ldots, x_{n}$ which, however, is a $\left(\frac{2}{\beta}\right)^{n}$-multiple of the element (16), plus a $\mathbb{Z}_{2}\left[\beta, \frac{2}{\beta}\right]$-linear combination of the elements (15), and thus can be eliminated. On the other hand, the elements (15), (16) are $\mathbb{Z}_{2}\left[\beta, \frac{2}{\beta}\right]$-linearly independent in $P$ since (15) and $x_{1} \ldots x_{n}$ are $\mathbb{Q}_{2}\left[\beta, \frac{2}{\beta}\right]$-linearly independent in $\widetilde{Q}$.

Now we have proved that

$$
M=\bigoplus_{\substack{1 \leq k \leq n \\ 1 \leq i_{1}<\cdots<i_{k} \leq n}} \mathbb{Z}\left[\frac{\beta}{2}\right] / \mathbb{Z}[\beta] y_{i_{1} \ldots i_{k}}
$$

where the element $y_{i_{1} \ldots i_{k}}$ is in Tor-degree $k$ and dimension $2 k-2$.

Proposition 8. The elements $y_{i_{1} \ldots i_{k}}$ can be chosen in such a way that in $Q$, we have

$$
y_{i_{1} \ldots i_{k}}=\frac{\beta^{k}}{2}\left(\prod_{s=1}^{k}\left(1+\frac{\beta}{2} x_{i_{s}}\right)-1\right) \prod_{u=1}^{n-k}\left(2+\beta x_{j_{u}}\right)
$$

where $\left\{i_{1}, \ldots, i_{k}, j_{1}, \ldots, j_{n-k}\right\}=\{1, \ldots, n\}$.

Proof. An induction on $n$. For $n=1$, we know from [6] that

$$
y_{1}=\frac{\beta^{2}}{4} x_{1}=\frac{\beta}{2}\left(\left(1+\frac{\beta}{2} x_{1}\right)-1\right) \text {. }
$$


Now for $k<n$, the element $y_{i_{1} \ldots i_{k}}$ can be chosen as a restriction from $k_{*} B(\mathbb{Z} / 2)^{k}$ for some subgroup of $(\mathbb{Z} / 2)^{n}$ isomorphic to $(\mathbb{Z} / 2)^{k}$. Thus, in $Q$, it will be given by corestriction of the same element from the same subgroup, which is given by multiplication by

$$
\prod_{u=1}^{n-k}\left(2+\beta x_{j_{u}}\right) \text {. }
$$

Hence, our statement for $y_{i_{1} \ldots i_{k}}, k<n$, follows from the induction hypothesis. For $k=n$, on the other hand, we can choose

$$
y_{i_{1} \ldots i_{n}}=-\frac{\beta^{n}}{2}
$$

simply by its dimension and the fact that $Q \subset \widetilde{Q}$ is a submodule (and thus, in particular, free of torsion in $\beta$ and 2).

Thus, it remains to calculate the extension determined by the relations (18). To this end, it is helpful to change variables by putting

$$
t_{i}=x_{i}+\frac{2}{\beta} \text {. }
$$

Lemma 9. The $\mathbb{Z}_{2}\left[\beta, \frac{2}{\beta}\right]$-module $P$ is free on the generators

$$
\begin{aligned}
& z_{i_{1} \ldots i_{k}}=t_{i_{1}} \ldots t_{i_{k}}-\left(\frac{2}{\beta}\right)^{k}, 1 \leq k<n, 1 \leq i_{1}<\cdots<i_{k} \leq n \\
& z_{1 \ldots n}=\left(\frac{\beta}{2}\right)^{n} t_{1} \ldots t_{n}-1 .
\end{aligned}
$$

Proof. The base change matrix between the generators (20) and those of Proposition 7 is triangular with invertible elements on the diagonal.

Proposition 10. The generators $y_{i_{1} \ldots i_{k}}$ of Proposition 8 can be changed to generators $y_{i_{1} \ldots i_{k}}^{\prime}$ such that in $Q$, we have

$$
y_{i_{1} \ldots i_{k}}^{\prime}=\frac{\beta^{n}}{2} \cdot z_{i_{1}, \ldots i_{k}}, 1 \leq k \leq n, 1 \leq i_{1}<\cdots<i_{k} \leq n .
$$

Proof. By Proposition 8, applying the base change, we have in $Q$ :

$$
y_{i_{1} \ldots i_{k}}=\frac{\beta^{n}}{2} \cdot\left(\left(\frac{\beta}{2}\right)^{k} \prod_{s=1}^{k} t_{i_{s}}-1\right) \prod_{u=1}^{n-k} t_{j_{u}} .
$$


For $k=n$, this is the desired generator. For $1 \leq k<n$, we get the desired generator by subtracting $y_{i_{1} \ldots i_{k}}$ from

$$
\frac{\beta^{n}}{2}\left(\frac{2}{\beta}\right)^{n-k}\left(\left(\frac{\beta}{2}\right)^{n} \prod_{s=1}^{n} t_{s}-1\right),
$$

which is in the image of $\left(\frac{2}{\beta}\right)^{n-k} y_{1 \ldots n}$. (Note that for $1 \leq k<n$, the sets $\left\{i_{1}<\cdots<i_{k}\right\}$ are in bijective correspondence with their complements.)

Let $Q_{n}$ be the $\mathbb{Z}_{2}\left[\beta, \frac{2}{\beta}\right]$-submodule of $\mathbb{Q}_{2}\left[\beta, \beta^{-1}\right]$ generated by $1, \beta^{n-1}\left(\frac{\beta}{2}\right)^{i}$, $i \in \mathbb{N}$. Then, as remarked, we have a unique $k$-module $\mathscr{Q}_{n}$ with coefficients $Q_{n}$. We have proved

Theorem 11. For $n \geq 0$, the Tate cohomology (11) is isomorphic, as a $k$-module, to

$$
\mathscr{Q}_{n} \cdot\left(\left(1+w^{-2}\right)^{n}-w^{-2 n}\right) \vee H \cdot f(w)
$$

where

$$
\begin{aligned}
& f(w)=\frac{w}{\left(1-w^{2}\right)^{2}(1-w)^{n-1}}\left(\frac{1-\left(1+w^{2}\right)^{n}(1-w)^{n}}{1-\left(1+w^{2}\right)(1-w)}-\frac{1-(1-w)^{n}}{1-(1-w)}\right)+ \\
& \frac{1}{\left(1-w^{-2}\right)^{2}\left(1-w^{-1}\right)^{n-1}}\left(\frac{1-\left(1+w^{-2}\right)^{n}\left(1-w^{-1}\right)^{n}}{1-\left(1+w^{-2}\right)\left(1-w^{-1}\right)}-\frac{1-\left(1-w^{-1}\right)^{n}}{1-\left(1-w^{-1}\right)}\right)
\end{aligned}
$$

The structure of $\mathscr{Q}_{n}$ as a spectrum is clarified by the following

Proposition 12. As spectra, we have:

$$
\begin{gathered}
k_{2}^{\wedge}\left[\frac{2}{\beta}\right]=k_{2}^{\wedge} \vee \bigvee_{n \geq 1} H \mathbb{Z}_{2}[-2 n], \\
k_{2}^{\wedge}\left[\frac{2}{\beta}, \frac{\beta}{2}\right]=\bigvee_{n \in \mathbb{Z}} H \mathbb{Z}_{2}[2 n], \\
\mathscr{Q}_{n}=\bigvee_{i \geq 1} H \mathbb{Z}_{2}[-2 i] \vee \tau_{\leq 2 n-2} k_{2}^{\wedge} \vee \bigvee_{i \geq 0} H \mathbb{Z}_{2}[2 n+2 i]
\end{gathered}
$$

where $\tau_{\leq m} X$ of a spectrum $X$ denotes the part of the Postnikov tower up to and including the $m$ 'th homotopy group. 
Proof. For (21), we have a map of $k$-modules

$$
k_{2}^{\wedge}\left[\frac{2}{\beta}\right] \rightarrow\left(k_{2}^{\wedge} \vee \bigvee_{n \geq 1} H \mathbb{Z}[-2 n]\right) \wedge M \mathbb{Z} / 2,
$$

mapping to each summand by killing the other generators. In spectra, the map (24) survives the $d_{1}$ of the corresponding Bockstein spectral sequence, which has no higher differentials (since the $E_{2}$-term is concentrated in even degrees), thus proving (21). Then (22) follows by localization. For (23), it then suffices to remark that we have a homotopy pushout of $k$-modules (and hence spectra)

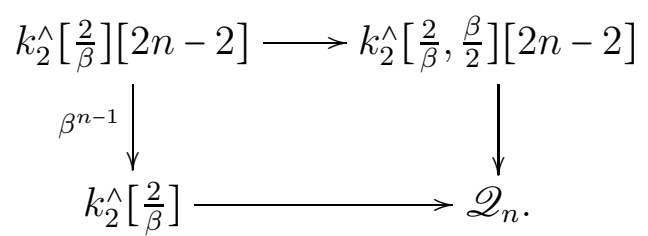

\section{The CASE of $p>2$}

The case of $G=(\mathbb{Z} / p)^{n}$ for $p>2$ prime is directly analogous (or more precisely a direct generalization). Nevertheless, it still introduces some new effects. For one thing, while not necessary, it is convenient to localize at $p$, which is possible in the category of $E_{\infty}$-ring spectra, so we have an $E_{\infty}$-ring spectrum $k_{(p)}$. We may start out with this spectrum, since the Tate cohomology is $p$-complete anyway. The reason it is convenient to localize is that then we can make the multiplicative group $p$-typical, with $p$-series

$$
[p] x=p x+\beta^{p-1} x^{p}
$$

(where $[p] x$ denotes the $p$-series). For Borel homology and cohomology, the basic building blocks are the $\mathbb{Z}_{(p)}[\beta]$-modules

$$
\begin{gathered}
N=\mathbb{Z}_{(p)}\left[\beta, \frac{\beta^{p-1}}{p}\right], \\
M=\left(N / \mathbb{Z}_{(p)}[\beta]\right)[2(2-p)], \\
\widetilde{H}=\mathbb{Z}_{(p)}[\beta] /\left(p, \beta^{p-1}\right) .
\end{gathered}
$$

The reason for the shift is that that way, the bottom degree element is in dimension 2, and other summands we encounter are shifts in the positive dimension, which seems more natural.

The modules $M, N$ have resolutions in degrees 0,1 , so they are uniquely realized by $k_{(p)}$-modules $\mathscr{M}, \mathscr{N}$. The module $\widetilde{H}$ is realized by 
the $k_{(p)}$-module $\widetilde{\mathscr{H}}=k_{(p)} /\left(p, \beta^{p-1}\right)$ (i.e. by killing a regular sequence). We then have

$$
k_{(p)} \wedge B \mathbb{Z} / p_{+}=k_{(p)} \vee \mathscr{M}[-1]\left(1+w^{2}+\ldots w^{2(p-2)}\right) .
$$

Directly analogously to Lemma 1, one proves

Lemma 13. (1) We have

$$
N \otimes_{\mathbb{Z}_{(p)}[\beta]} N \cong N \oplus \widetilde{H} \frac{w^{2(p-1)}}{\left(1-w^{2(p-1)}\right)^{2}}, \operatorname{Tor}_{1}(N, N)=0 .
$$

(2) We have

$$
N \otimes_{\mathbb{Z}_{(p)}[\beta]} M \cong \widetilde{H} \frac{w^{2(p-1)}}{\left(1-w^{2(p-1)}\right)^{2}}, \operatorname{Tor}_{1}(M, N)=0 .
$$

(3) We have

$$
M \otimes_{\mathbb{Z}_{(p)}[\beta]} M \cong \widetilde{H} \frac{w^{4}}{\left(1-w^{2(p-1)}\right)^{2}}, \operatorname{Tor}_{1}(M, M) \cong M[2] .
$$

On $k_{(p)}$-modules, one then gets

$$
\begin{gathered}
\mathscr{M} \wedge_{k_{(p)}} \mathscr{M}=\mathscr{M}[3] \vee \widetilde{\mathscr{H}} \frac{w^{4}}{\left(1-w^{2(p-1)}\right)^{2}}, \\
\mathscr{M} \wedge_{k_{(p)}} \widetilde{\mathscr{H}}=\widetilde{\mathscr{H}} \frac{w^{2}(1+w)}{1-w^{2(p-1)}} .
\end{gathered}
$$

Using this, putting

$$
k_{(p)} \wedge B(\mathbb{Z} / p)_{+}^{i}=k_{(p)} \vee \mathscr{M} p_{i} \vee \widetilde{\mathscr{H}} q_{i},
$$

one has $p_{1}=w^{-1}\left(1+w^{2}+\cdots+w^{2(p-2)}\right), q_{1}=0$, and computes

$$
\begin{aligned}
& \left.\left(k_{(p)} \vee \mathscr{M} p_{i} \vee \widetilde{\mathscr{H}} q_{i}\right)\right) \wedge_{k_{(p)}}\left(k_{(p)} \vee \mathscr{M} w^{-1}\left(1+w^{2}+\cdots+w^{2(p-2)}\right)\right)= \\
& k_{(p)} \vee \mathscr{M}\left(\left(1+w^{2}+\cdots+w^{2(p-1)}\right) p_{i}+w^{-1}\left(1+w^{2}+\cdots+w^{2(p-2)}\right)\right) \vee \\
& \widetilde{\mathscr{H}}\left(p_{i} \frac{w^{3}}{\left(1-w^{2(p-1)}\right)\left(1-w^{2}\right)}+\frac{q_{i}}{1-w}\right) .
\end{aligned}
$$

This gives

$$
\begin{aligned}
p_{i} & =\left(\left(1+w^{2}+\cdots+w^{2(p-1)}\right)^{i}-1\right) w^{-3}, \\
q_{i+1} & =\frac{\left(1+w^{2}+\cdots+w^{2(p-1)}\right)^{i}-1}{\left(1-w^{2(p-1)}\right)\left(1-w^{2}\right)}+\frac{q_{i}}{1-w} .
\end{aligned}
$$

Solving the recursion, we obtain 
Proposition 14. We have

$$
\begin{aligned}
& k_{(p)} \wedge B(\mathbb{Z} / p)_{+}^{i}=k_{(p)} \vee \mathscr{M} w^{-3}\left(\left(1+w^{2}+\cdots+w^{2(p-1)}\right)^{i}-1\right) \vee \\
& \widetilde{\mathscr{H}} \frac{1}{\left(1-w^{2(p-1)}\right)\left(1-w^{2}\right)(1-w)^{i-1}} . \\
& \left(\frac{1-\left(1+w^{2}+\cdots+w^{2(p-1)}\right)^{i}(1-w)^{i}}{1-\left(1+w^{2}+\cdots+w^{2(p-1)}\right)(1-w)}-\frac{1-(1-w)^{i}}{1-(1-w)}\right) .
\end{aligned}
$$

The situation in Borel cohomology is again analogous. We have

$$
F\left(B \mathbb{Z} / p, k_{(p)}\right)=\mathscr{P}\left(1+w^{-2}+\cdots+w^{-2(p-2)}\right)
$$

where

$$
\mathscr{P}=x k_{(p)}\left[\left[x^{p-1}\right]\right] /\left((x \beta)^{p-1}+p\right) .
$$

Again, we have

$$
\mathscr{P} \widehat{\wedge}_{k_{(p)}} \mathscr{P}=\mathscr{P}[-2] \vee \widetilde{\mathscr{H}} \frac{w^{-4}}{\left(1-w^{2(p-1)}\right)^{2}}
$$

and

$$
\mathscr{P} \widehat{\wedge}_{k_{(p)}} \widetilde{\mathscr{H}}=\widetilde{\mathscr{H}} \frac{w^{-1}\left(1+w^{-1}\right)}{1-w^{-2(p-1)}},
$$

Putting these together, we obtain the following result.

Proposition 15. We have

$$
\begin{aligned}
& F\left(B(\mathbb{Z} / p)^{i}, k_{(p)}\right)=\mathscr{P} w^{-1}\left(\left(1+w^{-2}+\cdots+w^{-2(p-1)}\right)^{i}-1\right) \vee \\
& \widetilde{\mathscr{H}} \frac{1}{\left(1-w^{-2(p-1)}\right)\left(1-w^{-2}\right)\left(1-w^{-1}\right)^{i-1}} . \\
& \left(\frac{1-\left(1+w^{-2}+\cdots+w^{-2(p-1)}\right)^{i}\left(1-w^{-1}\right)^{i}}{1-\left(1+w^{-2}+\cdots+w^{-2(p-1)}\right)\left(1-w^{-1}\right)}-\frac{1-\left(1-w^{-1}\right)^{i}}{1-\left(1-w^{-1}\right)}\right) .
\end{aligned}
$$

To calculate Tate cohomology, we use again the cofibration sequence

$$
k_{(p)} \wedge B(\mathbb{Z} / p)_{+}^{n} \stackrel{N}{\longrightarrow} F\left(B(\mathbb{Z} / p)_{+}^{n}, k_{(p)}\right) \longrightarrow \widehat{k}^{(\mathbb{Z} / p)^{n}}
$$

together with the input from Proposition 14, 15. Again, for dimensional reasons, there is no room for extensions involving the copies of $\widetilde{\mathscr{H}}$. 
Thus, again, it suffices to compute the image $Q$ of $\widehat{k}^{(\mathbb{Z} / p)^{n}}$ in

$$
\widetilde{Q}=\widehat{K}^{(\mathbb{Z} / p)^{n}}=J_{p}^{\wedge}\left[\beta, \beta^{-1}\right]\left[\frac{1}{p}\right]=\bigoplus_{\substack{k \geq 1 \\ 1 \leq i_{1}<\cdots<i_{k} \leq n \\ 1 \leq \epsilon_{i} \leq p-1}} \mathbb{Q}_{p}\left[\beta, \beta^{-1}\right]\left\{x_{i_{1}}^{\epsilon_{1}} \ldots x_{i_{k}}^{\epsilon_{k}}\right\}
$$

where $J$ is, again, the augmentation ideal of $R(\mathbb{Z} / p)^{n}$. Again, this is also equal to

$$
\begin{aligned}
& K^{*}\left((B \mathbb{Z} / p)^{n}\right) / K_{*} \cdot \prod_{i=1}^{n}\left(p-\left(\beta x_{i}\right)^{p-1}\right)= \\
& \mathbb{Z}\left[\beta, \beta^{-1}\right]\left[\left[x_{1}, \ldots, x_{n}\right]\right] /\left(x_{i}\left(p+\left(\beta x_{i}\right)^{p-1}\right), \prod_{i=1}^{n}\left(p+\left(\beta x_{i}\right)^{p-1}\right)\right) .
\end{aligned}
$$

Again, $Q$ is a $k_{(p)}^{*}\left(B(\mathbb{Z} / p)^{n}\right)$-submodule of $(26)$, while on a multiple of $x_{i}, x_{i}^{p-1}=-\frac{p}{\beta^{p-1}}$. Thus, we have proved

Lemma 16. $Q$ is a $\mathbb{Z}_{p}\left[\beta, \frac{p}{\beta^{p-1}}\right]$-submodule of $\widetilde{Q}$.

Let again $P \subset Q$ be the image of $k_{(p)}^{*} B(\mathbb{Z} / p)^{n}$. Then we have an extension of $\mathbb{Z}\left[\beta, \frac{p}{\beta^{p-1}}\right]$-modules

$$
0 \rightarrow P \rightarrow Q \rightarrow M \rightarrow 0 .
$$

Analogously to Proposition [7, we then have

Proposition 17. The $\mathbb{Z}_{p}\left[\beta, \frac{p}{\beta^{p-1}}\right]$-module $P$ is free on generators

$$
\begin{aligned}
& x_{i_{1}}^{\epsilon_{1}} \ldots x_{i_{k}}^{\epsilon_{k}} x_{j_{1}}^{p-1} \ldots x_{j_{n-k}}^{p-1}, 1 \leq k<n, 0 \leq \epsilon_{s} \leq p-2 \text { for } 1 \leq s \leq k, \\
& i_{1}<\cdots<i_{k}, j_{1}<\cdots<j_{n-k}, \\
& \left\{i_{1}, \ldots, i_{k}, j_{1}, \ldots, j_{n-k}\right\}=\{1, \ldots, n\}, \\
& \quad\left(\prod_{i=1}^{n}\left(1+\frac{\beta^{p-1}}{p} x_{i}^{p-1}\right)-1\right) x_{i_{1}}^{i_{1}} \ldots x_{i_{k}}^{\epsilon_{k}}, 1 \leq k \leq n, 0 \leq \epsilon_{s} \leq p-2 .
\end{aligned}
$$

Now we have proved that

$$
M=\bigoplus_{\substack{1 \leq k \leq n \\ 1 \leq i_{1}<\cdots<i_{k} \leq n \\ 0 \leq \epsilon_{s} \leq p-2}} \mathbb{Z}\left[\frac{\beta^{p-1}}{p}\right] / \mathbb{Z}[\beta]\left\{y_{i_{1} \ldots i_{k}} x_{i_{1}}^{\epsilon_{1}} \ldots x_{i_{k}}^{\epsilon_{k}}\right\}
$$

where the generator $y_{i_{1} \ldots i_{k}}$ is in Tor-degree $k$ and dimension $(2 k-2)(p-$ 1). Analogously to Proposition 8 , one has 
Proposition 18. The elements $y_{i_{1} \ldots i_{k}}$ can be chosen in such a way that in $Q$, we have

$$
y_{i_{1} \ldots i_{k}}=\frac{\beta^{k(p-1)}}{2}\left(\prod_{s=1}^{k}\left(1+\frac{\beta^{p-1}}{p} x_{i_{s}}^{p-1}\right)-1\right) \prod_{u=1}^{n-k}\left(p+\beta x_{j_{u}}^{p-1}\right)
$$

where $\left\{i_{1}, \ldots, i_{k}, j_{1}, \ldots, j_{n-k}\right\}=\{1, \ldots, n\}$.

Now put

$$
t_{i}=x_{i}^{p-1}+\frac{p}{\beta^{p-1}} .
$$

The analogue of Lemma 9 is

Lemma 19. The $\mathbb{Z}_{p}\left[\beta, \frac{p}{\beta^{p-1}}\right]$-module $P$ is free on the generators

$$
\begin{aligned}
& z_{i_{1} \ldots i_{k}} x_{i_{1}}^{\epsilon_{1}} \ldots x_{i_{k}}^{\epsilon_{k}}=\left(t_{j_{1}} \ldots t_{j_{n-k}}-\left(\frac{p}{\beta^{p-1}}\right)^{k}\right) x_{i_{1}}^{\epsilon_{1}} \ldots x_{i_{k}}^{\epsilon_{k}}, \\
& 1 \leq k<n, 1 \leq i_{1}<\cdots<i_{k} \leq n, 0 \leq \epsilon_{s} \leq p-2 \text { for } 1 \leq s \leq k \\
& j_{1}<\cdots<j_{n-k},\left\{i_{1}, \ldots, i_{k}, j_{1}, \ldots, j_{n-k}\right\}=\{1, \ldots, n\} \\
& z_{1 \ldots n} x_{1}^{\epsilon_{1}} \ldots x_{n}^{\epsilon_{n}}=\left(\left(\frac{\beta^{p-1}}{p}\right)^{n} t_{1} \ldots t_{n}-1\right), 0 \leq \epsilon_{s} \leq p-2
\end{aligned}
$$

Now analogously to Proposition 10, we have

Proposition 20. The generators $y_{i_{1} \ldots i_{k}}$ of Proposition 8 can be changed to generators $y_{i_{1} \ldots i_{k}}^{\prime}$ such that in $Q$, we have

$$
y_{i_{1} \ldots i_{k}}^{\prime}=\frac{\beta^{n(p-1)}}{p} \cdot z_{i_{1} \ldots i_{k}}, 1 \leq k \leq n, 1 \leq i_{1}<\cdots<i_{k} \leq n .
$$

Let $Q_{n}$ be the $\mathbb{Z}_{p}\left[\beta, \frac{p}{\beta^{p-1}}\right]$-submodule of $\mathbb{Q}_{p}\left[\beta, \beta^{-1}\right]$ generated by $1, \beta^{(p-1)(n-1)}\left(\frac{\beta^{p-1}}{p}\right)^{i}, i \in \mathbb{N}$. Then, as remarked, we have a unique $k$ module $\mathscr{Q}_{n}$ with coefficients $Q_{n}$. We have proved

Theorem 21. For $n \geq 0$, the Tate cohomology $\widehat{k}^{(\mathbb{Z} / p)^{n}}$ is isomorphic, as a $k$-module, to

$$
\mathscr{Q}_{n} \cdot\left(\left(1+w^{-2}+\cdots+w^{-2(p-1)}\right)^{n}-w^{-2 n(p-1)}\right) \vee \widetilde{\mathscr{H}} \cdot f(w)
$$


where

$$
\begin{aligned}
& f(w)=\frac{w}{\left(1-w^{2(p-1)}\right)\left(1-w^{2}\right)(1-w)^{n-1}} \\
& \left(\frac{1-\left(1+w^{2}+\cdots+w^{2(p-1)}\right)^{n}(1-w)^{n}}{1-\left(1+w^{2}+\cdots+w^{2(p-1)}\right)(1-w)}-\frac{1-(1-w)^{n}}{1-(1-w)}\right)+ \\
& \frac{1}{\left(1-w^{-2(p-1)}\right)\left(1-w^{-2}\right)\left(1-w^{-1}\right)^{n-1}} . \\
& \left(\frac{1-\left(1+w^{-2}+\cdots+w^{-2(p-1)}\right)^{n}\left(1-w^{-1}\right)^{n}}{1-\left(1+w^{-2}+\cdots+w^{-2(p-1)}\right)\left(1-w^{-1}\right)}-\frac{1-\left(1-w^{-1}\right)^{n}}{1-\left(1-w^{-1}\right)}\right) .
\end{aligned}
$$

As spectra, analogously to Proposition [12, one sees that $\widetilde{\mathscr{H}}$ is a wedge of suspensions of $H \mathbb{Z} / p$, while, denoting by $\ell$ the Adams summand of $k_{p}^{\wedge}, \mathscr{Q}_{n}$ is

$$
\bigvee_{i \geq 1} H \mathbb{Z}_{p}[-2 i] \wedge \tau_{\leq 2(p-1)(n-1)} k_{p}^{\wedge} \vee \bigvee_{i \geq 0} H \mathbb{Z}_{p}[2(p-1) n+2 i]
$$

The spectrum $\tau_{\leq 2(p-1)(n-1)} k_{p}^{\wedge}$, of course, further decomposes as a wedge of suspensions of $\tau_{\leq 2(p-1)(n-1)} \ell$ where $\ell$ is the Adams summand of $k_{p}^{\wedge}$.

\section{Relation with the Calculation of Bruner and GREENLEES}

In this Section, we prove that our computation of Borel homology, Borel cohomology and Tate cohomology for $p=2$ agrees with the result of Bruner and Greenlees [2]. In fact, the only non-trivial part left concerns the coefficient of the $H$ summand in Proposition 4, part 3 for Borel homology, Proposition 5 for Borel cohomology, and Theorem 11 for Tate cohomology. In fact, it suffices to compute the case of Borel homology, since both [2] and our paper have a duality which determines the other two cases in the same way.

Bruner and Greenlees [2] define

$$
\left[T_{i}\right]=\frac{(-t)^{4-i}\left[(1-x)_{[i]}^{r}-x^{1-i}\left(1-x^{2}\right)_{[i]}^{r}\right]}{(1-x)^{r+1}}
$$

$x=t^{2}$. By [2], Lemma 4.8.3, the Poincare series of the coefficients of the $H$-summand of $k \wedge B(\mathbb{Z} / 2)_{+}^{r}$, in the variable $t$, is

$$
\left[T_{2}\right]+\left[T_{3}\right]+\cdots+\left[T_{r}\right]
$$


We will separately compute

$$
\sum_{i=2}^{r}(-t)^{4-i}(1-x)_{[i]}^{r}
$$

and

$$
\sum_{i=2}^{r}(-t)^{4-i} x^{1-i}\left(1-x^{2}\right)_{[i]}^{r}
$$

For formula (34), we have $\left(\begin{array}{l}r \\ i\end{array}\right)$ occurring in $\left[T_{2}\right], \ldots,\left[T_{i}\right]$. For $2 \leq j \leq i$, the term in $\left[T_{j}\right]$ is

$$
(-t)^{4-j}\left(\begin{array}{l}
r \\
i
\end{array}\right)(-x)^{i}=(-1)^{j} t^{4-j}\left(\begin{array}{l}
r \\
i
\end{array}\right)(-1)^{i} t^{2 i}=\left(\begin{array}{l}
r \\
i
\end{array}\right)(-1)^{i+j} t^{2 i+4-j}
$$

Thus, letting $j$ run from 2 to $i$, the coefficient of $\left(\begin{array}{l}r \\ i\end{array}\right)$ in (34) is $t^{i+4}-t^{i+5}+\cdots+(-1)^{i-2} t^{2 i+2}=t^{i+4}\left(1-t+t^{2}-\cdots+(-1)^{i-2} t^{i-2}\right)=t^{i+4} \cdot \frac{1-(-t)^{i-1}}{1+t}$.

Now sum over $i=2, \ldots, r$, we get (34) is

$$
\begin{aligned}
\frac{1}{1+t} \sum_{i=2}^{r}\left(\begin{array}{l}
r \\
i
\end{array}\right) t^{i+4}\left(1-(-t)^{i-1}\right) & =\frac{1}{1+t}\left[\sum_{i=2}^{r}\left(\begin{array}{l}
r \\
i
\end{array}\right) t^{i+4}-\sum_{i=2}^{r}\left(\begin{array}{l}
r \\
i
\end{array}\right)(-1)^{i-1} t^{2 i+3}\right] \\
& =\frac{1}{1+t}\left[t^{4} \sum_{i=2}^{r}\left(\begin{array}{l}
r \\
i
\end{array}\right) t^{i}+t^{3} \sum_{i=2}^{r}\left(\begin{array}{l}
r \\
i
\end{array}\right)(-1)^{i} t^{2 i}\right] \\
& =\frac{1}{1+t}\left[t^{4}\left[(1+t)^{r}-r t-1\right]+t^{3}\left[\left(1-t^{2}\right)^{r}-r\left(-t^{2}\right)-1\right]\right] \\
& =\frac{1}{1+t}\left[t^{4}(1+t)^{r}-r t^{5}-t^{4}+t^{3}\left(1-t^{2}\right)^{r}+r t^{5}-t^{3}\right] \\
& =\frac{1}{1+t}\left[t^{4}(1+t)^{r}-t^{4}+t^{3}\left(1-t^{2}\right)^{r}-t^{3}\right] .
\end{aligned}
$$

Hence, we get that formula (34) is

$$
\frac{t^{3}}{1+t}\left[t\left((1+t)^{r}-1\right)+\left(\left(1-t^{2}\right)^{r}-1\right)\right] .
$$

Now for formula (35), again, $\left(\begin{array}{c}r \\ i\end{array}\right)$ occurs in $\left[T_{2}\right], \ldots,\left[T_{i}\right]$. For $2 \leq j \leq i$, the term containing $\left(\begin{array}{l}r \\ i\end{array}\right)$ in $\left[T_{j}\right]$ is

$$
(-1)^{4-j} x^{1-j}\left(\begin{array}{l}
r \\
i
\end{array}\right)\left(-x^{2}\right)^{i}=(-1)^{j} t^{4-j} t^{2-2 j}\left(\begin{array}{l}
r \\
i
\end{array}\right)(-1)^{i} t^{4 i}=\left(\begin{array}{l}
r \\
i
\end{array}\right)(-1)^{i+j} t^{4 i-3 j+6}
$$


Summing over $2 \leq j \leq i$, we get the coefficient of $\left(\begin{array}{l}r \\ i\end{array}\right)$ in (35) is

$$
(-1)^{i} t^{4 i}+(-1)^{i-1} t^{4 i-3}+\cdots+t^{i+6} .
$$

(The lowest power of $t$ is always positive.) This is in turn

$$
t^{i+6}\left(1-t^{3}+t^{6}+\cdots+(-1)^{i} t^{3 i-6}\right)=t^{i+6} \cdot \frac{1-\left(-t^{3}\right)^{i-1}}{1+t^{3}}
$$

Hence, (35) is

$$
\begin{aligned}
& \frac{1}{1+t^{3}} \sum_{i=2}^{r}\left(\begin{array}{l}
r \\
i
\end{array}\right) t^{i+6}\left[1-\left(-t^{3}\right)^{i-1}\right] \\
& =\frac{1}{1+t^{3}}\left[\sum_{i=2}^{r}\left(\begin{array}{l}
r \\
i
\end{array}\right) t^{i+6}-\sum_{i=2}^{r}\left(\begin{array}{l}
r \\
i
\end{array}\right) t^{i+6}\left(-t^{3}\right)^{i-1}\right] \\
& =\frac{1}{1+t^{3}}\left[t^{6} \sum_{i=2}^{r}\left(\begin{array}{l}
r \\
i
\end{array}\right) t^{i}+\sum_{i=2}^{r}\left(\begin{array}{l}
r \\
i
\end{array}\right)(-1)^{i} t^{4 i+3}\right] \\
& =\frac{1}{1+t^{3}}\left[t^{6} \sum_{i=2}^{r}\left(\begin{array}{l}
r \\
i
\end{array}\right) t^{i}+t^{3} \sum_{i=2}^{r}\left(\begin{array}{l}
r \\
i
\end{array}\right)\left(-t^{4}\right)^{i}\right] \\
& =\frac{1}{1+t^{3}}\left[t^{6}\left[(1+t)^{r}-r t-1\right]+t^{3}\left[\left(1-t^{4}\right)^{r}-r\left(-t^{4}\right)-1\right]\right] \\
& =\frac{t^{3}}{1+t^{3}}\left[t^{3}\left((1+t)^{r}-1\right)+\left(\left(1-t^{4}\right)^{r}-1\right)\right] .
\end{aligned}
$$

Putting it together, we get that (33) is

$$
\frac{1}{\left(1-t^{2}\right)^{r+1}}\left[\begin{array}{c}
\frac{t^{3}}{1+t}\left[t\left((1+t)^{r}-1\right)+\left(\left(1-t^{2}\right)^{r}-1\right)\right] \\
-\frac{t^{3}}{1+t^{3}}\left[t^{3}\left((1+t)^{r}-1\right)+\left(\left(1-t^{4}\right)^{r}-1\right)\right]
\end{array}\right] .
$$

It seems better to set $u=-t$, so we get that (36) is

$$
\frac{1}{\left(1-u^{2}\right)^{r+1}}\left[\begin{array}{c}
\frac{u^{4}}{1-u}\left((1-u)^{r}-1\right)-\frac{u^{3}}{1-u}\left(\left(1-u^{2}\right)^{r}-1\right) \\
-\frac{u^{6}}{1-u^{3}}\left((1-u)^{r}-1\right)+\frac{u^{3}}{1-u^{3}}\left(\left(1-u^{4}\right)^{r}-1\right)
\end{array}\right]
$$

In (37), the two terms of the first column in the bracket add up to

$$
\left(\frac{u^{4}}{1-u}-\frac{u^{6}}{1-u^{3}}\right)\left[(1-u)^{r}-1\right]=\frac{u^{4}(1+u)}{1-u^{3}}\left[(1-u)^{r}-1\right] .
$$


The two terms of the second column inside the bracket of (37) add up to

$$
\begin{aligned}
& \frac{u^{3}}{1-u^{3}}\left[\left(1-u^{4}\right)^{r}-1\right]-\frac{u^{3}}{1-u}\left[\left(1-u^{2}\right)^{r}-1\right] \\
& =\frac{u^{3}}{1-u^{3}}\left[\left(1-u^{2}\right)^{r}\left(1+u^{2}\right)^{r}-1\right]-\frac{u^{3}\left(1+u+u^{2}\right)}{1-u^{3}}\left[\left(1-u^{2}\right)^{r}-1\right] \\
& =\frac{u^{3}}{1-u^{3}}\left[\left(1+u^{2}\right)^{r}-1\right]\left(1-u^{2}\right)^{r}-\frac{u^{4}(1+u)}{1-u^{3}}\left[\left(1-u^{2}\right)^{r}-1\right]
\end{aligned}
$$

Combining the second term of this with (38), we get

$$
\begin{aligned}
& -\frac{u^{4}(1+u)}{1-u^{3}}\left[(1-u)^{r}(1+u)^{r}-1-(1-u)^{r}+1\right] \\
& =-\frac{u^{4}(1+u)}{1-u^{3}}\left[(1+u)^{r}-1\right](1-u)^{r}
\end{aligned}
$$

Hence, (37) becomes

$$
\begin{aligned}
& \frac{1}{\left(1-u^{2}\right)^{r+1}}\left[\frac{u^{3}}{1-u^{3}}\left[\left(1+u^{2}\right)^{r}-1\right]\left(1-u^{2}\right)^{r}-\frac{u^{4}(1+u)}{1-u^{3}}\left[(1+u)^{r}-1\right](1-u)^{r}\right] \\
& =\frac{u^{3}}{1-u^{3}}\left[\frac{\left(1+u^{2}\right)^{r}-1}{1-u^{2}}-\frac{1}{\left(1-u^{2}\right)^{r+1}} u(1+u)\left[(1+u)^{r}-1\right](1-u)^{r}\right] \\
& =\frac{u^{3}}{1-u^{3}}\left[\frac{\left(1+u^{2}\right)^{r}-1}{1-u^{2}}-\frac{u\left[(1+u)^{r}-1\right]}{(1+u)^{r}(1-u)}\right] .
\end{aligned}
$$

If we put this back into $t$, we get that (33) is

$$
-\frac{t^{3}}{1+t^{3}}\left[\frac{\left(1+t^{2}\right)^{r}-1}{1-t^{2}}+\frac{t\left[(1-t)^{r}-1\right]}{(1-t)^{r}(1+t)}\right] .
$$

By Proposition 4, part 3, the coefficient of the $H$-summand of the Borel homology spectrum $k \wedge B(\mathbb{Z} / 2)_{+}^{r}$ is

$$
\frac{1}{\left(1-w^{2}\right)^{2}(1-w)^{r-1}}\left(\frac{1-\left(1+w^{2}\right)^{r}(1-w)^{r}}{1-\left(1+w^{2}\right)(1-w)}-\frac{1-(1-w)^{r}}{1-(1-w)}\right) .
$$

(We continue using $w$ instead of $t$ for the formula in our paper, to keep the notation distinct from that of Bruner-Greenlees.)

The Bruner-Greenlees formula needs to be applied with what they call Start(2), i. e. shift so that the lowest term is $t^{2}$, which matches what we get from formula (40). 
In fact, in Bruner-Greenlees [2], proof of Lemma 4.8.3, they compute explicitly

$$
\left(1-t^{2}\right)^{r}\left[T_{i}\right]=\left(\begin{array}{c}
r \\
i
\end{array}\right) t^{i+4}-\left(\begin{array}{c}
r \\
i+1
\end{array}\right)\left(t^{i+6}+t^{i+8}\right)+\left(\begin{array}{c}
r \\
i+2
\end{array}\right)\left(t^{i+8}+t^{i+10}+t^{i+12}\right)-\cdots
$$

So the lowest term in $\left[T_{2}\right]+\cdots\left[T_{r}\right]$ is always $\left(\begin{array}{l}r \\ 2\end{array}\right) t^{6}$ from $\left[T_{2}\right]$. Hence, Start(2) on it is just multiplying by $t^{-4}$. Applying this to (39), we get

$$
\frac{-1}{t\left(1+t^{3}\right)}\left[\frac{\left(1+t^{2}\right)^{r}-1}{1-t^{2}}+\frac{t\left[(1-t)^{r}-1\right]}{(1-t)^{r}(1+t)}\right] \text {. }
$$

Lemma 22. Formulas (40) and (41) agree (up to replacingt by $w$ ).

Proof. To show this, note that (41) is

$$
\begin{aligned}
& \frac{-1}{t\left(1+t^{3}\right)}\left[\frac{\left(1+t^{2}\right)^{r}-1}{1-t^{2}}+\frac{t\left[(1-t)^{r}-1\right]}{\left(1-t^{2}\right)(1-t)^{r-1}}\right] \\
& =\frac{-1}{t\left(1+t^{3}\right)}\left[\frac{\left[\left(1+t^{2}\right)^{r}-1\right](1-t)^{r-1}+t\left[(1-t)^{r}-1\right]}{\left(1-t^{2}\right)(1-t)^{r-1}}\right] \\
& =\frac{-1}{t\left(1-t+t^{2}\right)(1+t)}\left[\frac{\left[\left(1+t^{2}\right)^{r}-1\right](1-t)^{r-1}+t\left[(1-t)^{r}-1\right]}{\left(1-t^{2}\right)(1-t)^{r-1}}\right] \\
& =\frac{-1}{t\left(1-t+t^{2}\right)}\left[\frac{\left[\left(1+t^{2}\right)^{r}-1\right](1-t)^{r-1}+t\left[(1-t)^{r}-1\right]}{\left(1-t^{2}\right)^{2}(1-t)^{r-2}}\right] \\
& =\frac{-1}{t\left(1-t+t^{2}\right)}\left[\frac{\left[\left(1+t^{2}\right)^{r}-1\right](1-t)^{r}+t(1-t)\left[(1-t)^{r}-1\right]}{\left(1-t^{2}\right)^{2}(1-t)^{r-1}}\right] .
\end{aligned}
$$

On the other hand, for our formula (40), let $a=1+w^{2}, b=1-w$. The part inside the brackets of (40) is

$$
\frac{1-a^{r} b^{r}}{1-a b}-\frac{1-b^{r}}{1-b}=\frac{\left(1-a^{r} b^{r}\right)(1-b)-(1-a b)\left(1-b^{r}\right)}{(1-a b)(1-b)}
$$

The denominator is

$$
\begin{aligned}
& 1-\left(1+w^{2}\right)(1-w)-(1-w)+\left(1+w^{2}\right)(1-w)^{2} \\
& =\left(1+w^{2}\right)\left[(1-w)^{2}-(1-w)\right]+w \\
& =w^{4}-w^{3}+w^{2} \\
& =w^{2}\left(1-w+w^{2}\right) .
\end{aligned}
$$

Hence, (40) becomes

$$
\frac{1}{\left(1-w^{2}\right)^{2}(1-w)^{r-1}}\left(\frac{\left(1-a^{r} b^{r}\right)(1-b)-(1-a b)\left(1-b^{r}\right)}{w^{2}\left(1-w+w^{2}\right)}\right) .
$$


Comparing this to what we get from (41) above, it suffices to show that

$$
\frac{\left(1-a^{r} b^{r}\right)(1-b)-(1-a b)\left(1-b^{r}\right)}{w}
$$

is the same as

$$
\begin{aligned}
& -\left[\left[\left(1+t^{2}\right)^{r}-1\right](1-t)^{r}+t(1-t)\left[(1-t)^{r}-1\right]\right] \\
& =-\left[\left(1+t^{2}\right)^{r}(1-t)^{r}-(1-t)^{r}+t(1-t)\left[(1-t)^{r}-1\right]\right] \\
& =-\left(1+t^{2}\right)^{r}(1-t)^{r}+(1-t)^{r}-t(1-t)^{r+1}+t(1-t) .
\end{aligned}
$$

We have that the numerator of (43) is

$$
\begin{aligned}
& 1-a^{r} b^{r}-b+a^{r} b^{r+1}-\left(1-a b-b^{r}+a b^{r+1}\right) \\
& =-a^{r} b^{r}-b+a^{r} b^{r+1}+a b+b^{r}-a b^{r+1} \\
& =a^{r}\left(b^{r+1}-b^{r}\right)+(a-1) b+b^{r}(1-a b) .
\end{aligned}
$$

The first term of this is

$$
a^{r}\left(b^{r+1}-b^{r}\right)=a^{r} b^{r}(b-1)=-w\left(1+w^{2}\right)^{r}(1-w)^{r} .
$$

The second term is

$$
(a-1) b=w^{2}(1-w)
$$

The third term is

$$
\begin{aligned}
b^{r}(1-a b) & =(1-w)^{r}\left[1-\left(1+w^{2}\right)(1-w)\right] \\
& =(1-w)^{r}\left[w-w^{2}+w^{3}\right] \\
& =w(1-w)^{r}-w^{2}(1-w)^{r+1} .
\end{aligned}
$$

Taking the sum of (45), (46), (47) and dividing by $w$, we precisely get (44) when we equate $w$ and $t$.

\section{REFERENCES}

[1] M.F.Atiyah, G.B.Segal: Equivariant K-theory and completion, J. Diff. Geom. 3 (1969) 1-18

[2] R.R.Bruner, J.P.C.Greenlees: The connective K-theory of finite groups, Mem. Amer. Math. Soc. 165 (2003), no. 785, AMS, Providence, RI

[3] G.Carlsson: Equivariant stable homotopy and Segal's Burnside ring conjecture, Ann. of Math. 120 (1984) 189-224

[4] D.M.Davis, M.Mahowald: The spectrum $(P \wedge b o)_{-\infty}$, Math. Proc. Cambridge Phil. Soc. 96 (1984) 85-93

[5] A. Elmendorf, I. Kriz, M. Mandell, P. May: Rings, modules and algebras in stable homotopy theory, AMS Mathematical Surveys and Monographs, Volume 47, 1997 
[6] J.P.C.Greenlees, J.P.May: Generalized Tate cohomology, Memoirs AMS 543, 1995

[7] J.P.C.Greenlees, J.P.May: Localization and completion theorems for $M U$ module spectra, Ann. Math. 146 (1997) 509-544

[8] M. A. Hill, M. J. Hopkins, D. C. Ravenel: On the nonexistence of elements of Kervaire invariant one, Annals of Mathematics Volume 184 (2016), Issue 1, $1-262$

[9] P. Hu, I. Kriz: Real-oriented homotopy theory and an analogue of the AdamsNovikov spectral sequence, Topology 40(2) (2001) 317-399

[10] L. G. Lewis, Jr., J. P. May, M. Steinberger, and J. E. McClure: Equivariant stable homotopy theory, Vol 1213 of Lecture Notes in Mathematics, SpringerVerlag, Berlin, 1986.

[11] T.Nikolaus, P.Scholze: On topological cyclic homology, arXiv: 1707.01799

[12] J.-P.Serre: Cohomologie Galoisienne, Lecture Notes in Math. 5, Springer Verlag, 1994 